\title{
Progress in Compact Room Temperature THz Radiation Sources
}

Ross Leyman*, Natalia Bazieva, Tomasz Kruczek, Grigorii S. Sokolovskii, Edik U. Rafailov

Photonics \& Nanoscience Group, School of Engineering, Physics and Mathematics, University of Dundee, Dundee, DD1 4HN, United Kingdom

*r.r.leyman@dundee.ac.uk, Tel: +44 (0) 1382381 076, Fax: +44 (0) 1382388313

\begin{abstract}
There is currently a great deal of interest in the development of efficient compact sources of terahertz $(\mathrm{THz})$ radiation. There are many factors of device and materials design which are still under ongoing improvement, with the aim of producing an efficient, high power, room-temperature $\mathrm{THz}$ signal emission device which is tunable over a practical $\mathrm{THz}$ frequency range. The recent advances in production of various room-temperature $\mathrm{THz}$ radiation sources are reviewed with particular emphasis on photomixer antenna devices, and recent patents on technology and methods relating to: pulsed and continuous wave (CW) $\mathrm{THz}$ generation via nonlinear crystals; quantum cascade lasers (QCLs); ultrafast diodes and photodiodes; and heterodyne sources are discussed.
\end{abstract}

Keywords: $\mathrm{THz}$ radiation - generation and detection, photomixers, quantum dot (QD), semiconductor lasers, ultrashort pulse, dual-mode, nonlinear crystal, quantum cascade laser (QCL), resonant tunnelling diode (RTD), uni-travelling-carrier photodiode (UTC-PD). 


\section{INTRODUCTION}

Early work in $\mathrm{THz}$ optoelectronics was based on materials and devices driven by femtosecond optical pump signals generated by laser sources such as a Ti:Sapphire or ring-dye laser. These systems are now capable of performing time-domain spectroscopy and imaging in a range of applications including the security [1-3] and biomedical fields [4-6] and the demand for expansion of devices capable of analysing the information-rich $\mathrm{THz}$ region (typically defined as the region $0.3-10 \mathrm{THz}$ ) has led to the ongoing development of more compact, efficient and frequency-tunable $\mathrm{THz}$ sources. The so-called "terahertz gap" of the electromagnetic (EM) spectrum is located between the infrared and microwave regions and, because of its location, possesses a number of unique characteristics and properties. Spectroscopists began exploring the $\mathrm{THz}$ spectral region in the mid-20th century, and it is believed that they were first to apply the term "terahertz" to describe EM waves of this range, although its boundaries are rather blurred and subject to the field of application.

$\mathrm{THz}$ spectroscopy uncovers vast layers of valuable scientific information, which is almost impossible to extract any other way - it is a unique method of determining the spectra of a huge variety of light molecules and chemical compounds. $\mathrm{THz}$ high-resolution molecular spectroscopy makes it possible to study very diverse objects, from the biological to the cosmic scales - including the absorption and emission spectra of human tissue as well as stars and free space, which is rich with the energy of terahertz radiation. Recent years have brought the practical application of terahertz radiation far beyond the limits of research laboratories. Radiation in the terahertz range is non-ionizing, and it opens up expansive prospects for its application in medicine - for example, as implemented instead of X-rays in diagnostics. Timedomain spectroscopy, or "T-ray imaging", is at the moment a rapidly developing area of medical imaging. Despite the fact that the waves of this range are absorbed by water molecules, $\mathrm{THz}$ radiation is well established as a diagnostic method in dermatology, dentistry, etc. An excellent and detailed description of the traditional areas of application of terahertz radiation can be found in reference [7].

As the interest in $\mathrm{THz}$ optoelectronics research has grown, a wide variety of solutions have been presented. Development is still ongoing in crucial device properties such as: ultrafast electronic materials; electrooptic (EO) crystals; micro-antenna design; THz optics; optical pump sources; and the important aspect of detection. This review will look at recent patents and research which indicate promising advances in these areas specifically in the design of compact devices which generate $\mathrm{THz}$ signals operating at room temperature.

\section{METHODS OF THZ SIGNAL GENERATION}

Both pulsed and CW THz optoelectronics research stems from the same fundamental work into materials which display a component of nonlinear optical response in the $\mathrm{THz}$ frequency range 
[8-11], or which may electrically respond over ultra-short (a few picoseconds or shorter) timescales [12-16]. There have been many improvements in the efficiency of these systems, as well as several completely different approaches to the naturally difficult problem of generating signals within the THz gap. Predominant devices and methods of generating THz radiation to date include: exploitation of nonlinear effects in EO crystals such as ZnTe [17, 18] and periodically-poled $\mathrm{LiNbO}_{3}$ [19, 20]; emission from gas lasers [21-28]; QCL's [29-37]; high frequency microwave electronics such as Gunn diodes and so-called uni-travelling-carrierphotodiodes [38-42]; and photomixing techniques based on heterodyning of multiple optical pump signals [43-55]. There are many different approaches with a widely varying degree of success, but this review will focus specifically on compact, room temperature sources which have seen some considerable improvement in recent years - particularly with some emphasis on the highly active and expanding field of $\mathrm{THz}$ heterodyne devices. The figures of merit in the art are typically considered to be the optical-THz efficiency, the relative simplicity and dimensions and therefore the general practicality, the operating temperature, the achievable output power and the THz frequency tunability of the system or device. These aspects of device design will be the standards by which the recent progress in the field are considered in this review.

\section{Nonlinear Optical Materials}

Research in THz optoelectronic systems based on signals modulated via EO crystals has seen some considerable advances in device performance, efficiencies and achievable output power. Under optical excitation of a suitable wavelength which is efficiently absorbed by the medium, an EO crystal can undergo a change in electronic polarity within the volume which is excited. The high $\mathrm{X}^{(2)}$ nonlinear susceptibility of crystals such as $\mathrm{LiNb}_{3}$ allows the up-conversion of incoming EM waves as well as difference frequency generation (DFG, see later section) within the medium to achieve THz-range spectral transmission. The nonlinear susceptibility of commonly used EO crystals is used to generate both high power pulsed (i.e. optical rectification) $\mathrm{THz}$ output signals and tunable $\mathrm{CW} \mathrm{THz}$ output. In such materials, phase matching of the pump and generated signals becomes important to ensure that the generated $\mathrm{THz}$ wave is not interfered with destructively before it can be outcoupled to free space. If a practical degree of phase-matching can be achieved, the generated $\mathrm{THz}$ output power may be proportional to the pump power. This may be achieved in several ways, the most predominant of which are: by engineering of the birefringence of the crystal; by quasi-phase-matching (QPM) of propagating waves within the crystal; and by waveguiding.

Schulkin et al. in 2008 patented a THz spectroscopy device (including the design of the device housing) which is based on an EO crystal through which the components of EM polarisation of the pulsed pump and probe signals may be evaluated, and the resultant characterised $\mathrm{THz}$ radiation is passed through the target to be analysed which is placed in the 
housing in a small removable mount [56]. The target-modulated $\mathrm{THz}$ signal is then passed to another EO crystal for detection and analysis. This device has been marketed commercially under the title of the "Mini-Z", and is technically a compact, room-temperature source of $\mathrm{THz}$ radiation (as well as a $\mathrm{THz}$ detector) for use in a broad range of $\mathrm{THz}$ frequencies (particularly $0.01-5 \mathrm{THz}$ ), but this is because the device must be driven by an external pulsed pump source such as a Ti:Sapphire laser and the operating frequency is determined by the temporal pulsewidth of the external pump.

Moloney et al. recently developed a highly effective system which utilises externally pumped vertical cavity surface emitting lasers (VECSELs) as the pump sources and a periodically-poled $\mathrm{LiNb}_{3}$ (PPLN) crystal as the active medium, which was engineered with all of the aforementioned phase-control mechanisms integrated in the design of the crystal and included additional outcoupling measures at the crystal-air interface to further reduce losses [57]. In the setup (e.g. Figure 1), several configurations are employed in which the VECSELs are pumped externally and resonate with slightly offset optical wavelengths within an external cavity which the EO crystal is located. DFG of the VECSEL pumps occurs within the crystal, and the beating $\mathrm{THz}$ wave is propagated through the crystal volume enhanced by its phasematching properties, and is then outcoupled optically. The crystal birefringence (offset of refractive index between ordinarily and extraordinarily polarised propagating waves) is determined by the orientation of the crystal cleavage, and is chosen so to limit as far as possible the phase mismatch between the propagating ordinary and extraordinary EO signals. The crystal is periodically poled along the optical axis by applying a high electrical field across it, thereby creating opposite ferroelectric domains which typically vary periodically with the halfwavelength of the incoming pump signal. The inventors in this case have used this concept to

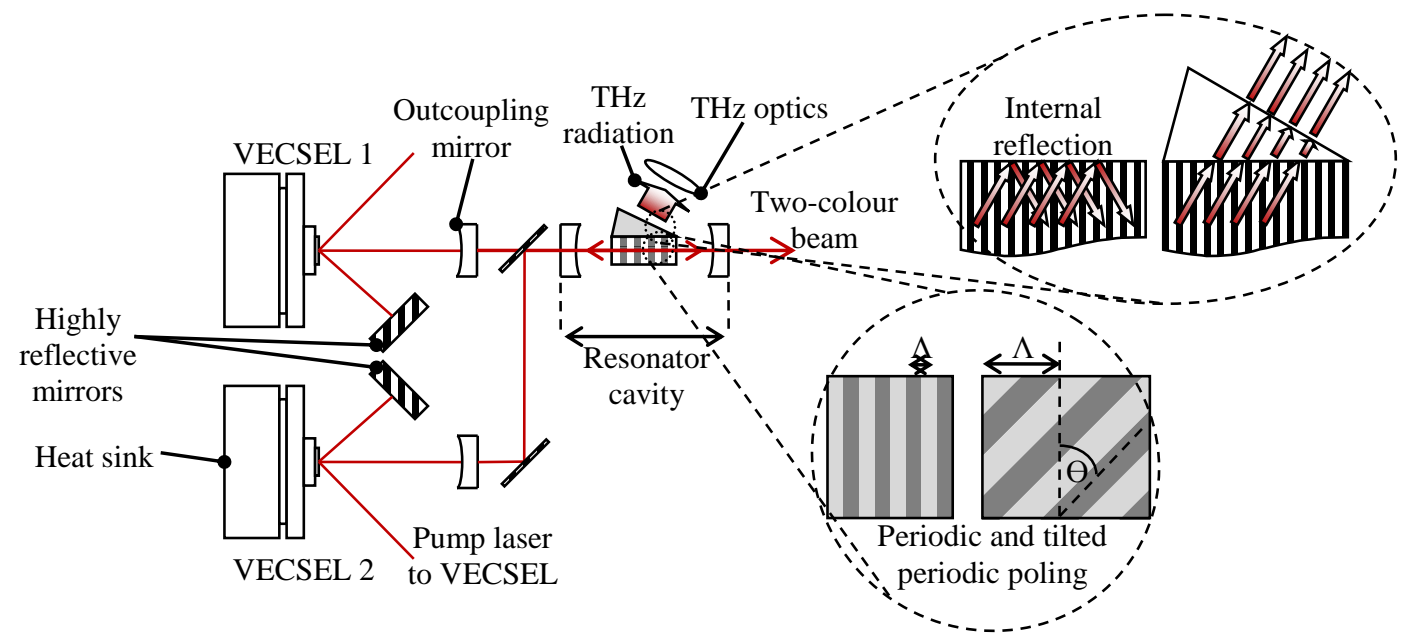

Fig. 1. Example optical schematic of the optimized VECSEL-pumped PPLN set up, with which Moloney et al. has patented a record high-power THz output, room temperature, $\mathrm{CW}$ system. The optimisations of the PPLN crystal itself are indicated in insets; (top right) with additional outcoupling cladding which prevents internal reflection at the crystal-air interface; and (bottom right) with a variety of tilted, periodic and aperiodic poling through the optical axis. 
develop a crystal with poling period(s) which correspond to the coherence length of both the pump and generated $\mathrm{THz}$ waves. This ensures that the generated nonlinear signal is not dampened by the propagating pump signal, as the phase "walk-off" between the two signals is periodically 'reset' towards zero, and the amplitude of the propagating $\mathrm{THz}$ signal is increased quadratically as the pump is driven through each successive periodic domain. The patent includes a discussion on the use of poled domains which vary alternatingly along the optical axis through the crystal, as well as the use of tilted and aperiodic poling domains (Figure 1, inset). Poling domains tilted at some angle $\Theta$ which corresponds to the direction of the $\mathrm{THz}$ wave propagation helps to compensate for the directional mismatch of the two propagating wavefront types and minimise the risk of destructive interference of the IR pump beam and the $\mathrm{THz}$ wave, and so the issue of divergence of the IR pump beam and subsequent reduction in the resultant $\mathrm{THz}$ output power is avoided. The tilt in the poling domains is calculated as $\tan (\theta)=$

$\frac{n_{T H z}}{n_{I R}}$; and the period of the poling is calculated as $\Lambda=\frac{\lambda_{T H z}}{n_{I R}} \cos (\theta)$, where $\mathrm{n}_{\mathrm{THz}}$ and $\mathrm{n}_{\mathrm{IR}}$ are the refractive indices of the crystal medium for the THz and IR pump waves respectively, $\lambda_{\mathrm{THz}}$ is the free-space $\mathrm{THz}$ wavelength and $\Lambda$ is the poling period. The crystal waveguide is structured so to achieve identical effective refractive indices in the nonlinear medium of both the IR pump signal and the $\mathrm{THz}$ generated signal, and is done so using known strip, ridge and slab waveguide structuring techniques. To reduce losses due to internal reflection at the crystal-air interface, an angled extension to the emission surface is included (Figure 1, inset) which significantly improves outcoupling efficiency.

This system is perhaps the most efficient THz radiation source based on an EO crystal as the emission medium to date. Reported $\mathrm{THz}$ output power is in the milliwatt and potentially up to the Watt range. The THz output frequency is also highly tunable: it is determined by the difference frequency of the two (VECSEL) optical signals, which is tuned simply by rotating an etalon or coupled diffraction grating to separate or bring together the two permitted pump wavelengths. The system is also relatively cost-effective, as high-power VECSELs and PPLN may be produced comparatively economically, and may have the dimensions suitable for practical, table-top use. The optical-THz efficiency is still rather low, however, with the cavity optical power being around 500W and some temperature control of the EO crystal required, despite including dopants such as $\mathrm{MgO}$ for thermal stability.

\section{Quantum Cascade Lasers}

QCL's were first proposed in 1971 [58] and have been implemented since 1994 [29] as a semiconductor laser source for generating pulsed and CW signals with wavelengths between around $2.5 \mu \mathrm{m}$ and $350 \mu \mathrm{m}$. This is achieved by stimulating emission from transitions within only the conduction band of a superlattice of thin semiconductor layers rather than by stimulating 
radiative recombination of carriers from both the conduction and valence bands, by which method a 'traditional' semiconductor laser would typically stimulate emission at higher energy, visible wavelengths. So, in comparison with a diode laser in which the bandgap essentially defines the emission wavelength(s), the energy levels of carriers in a QCL are determined by the extent of their quantum confinement within adjacent nano-scale layers throughout the superlattice, and the associated inter-layer wavefunction is used to determine the nature of the carriers' radiative transitions. A sloped potential is applied across the whole superlattice, electrons are injected into an excited level of the active layer's first well and a population inversion may be achieved as they tunnel through successive layers and relax radiatively into the lower state typically of the next layer, after which is the next injection layer and the process continues throughout the active region [29]. These processes are referred to as inter-subband transitions, and it is found that the upper lasing energy levels in thin quantum layers may be engineered to correspond with $\mathrm{THz}$ emission wavelengths.

Considerable effort in the field is dedicated to engineering of the upper and lower laser levels across the heterostructure of each active "module" (an active lasing heterostructure module is repeated many times through the QCL) so to achieve a rapid depopulation of the lower lasing level into the relaxation state and to maintain a reserve of carriers in the upper lasing level for efficient radiative relaxation. To this end, the lower lasing state sub-band may be engineered with an energy gap above the relaxation state which corresponds to the longitudinaloptical (LO) phonon energy of the layer material, which initiates so-called 'resonant phonon (RP) scattering' processes (Figure 2). Because of the reliance on phonon interaction, it is inherently difficult to operate a THz QCL efficiently without considerable device cooling (the upper temperature limit is currently $178 \mathrm{~K}$ ) and this is the subject of ongoing RP QCL research by groups such as Hu \& Williams [59]. Interestingly, QCL superlattice device structures may be subband-engineered to exhibit both lasing gain and very large $X^{(2)}$ susceptibility [60-65], and Belkin et al. have developed a QCL concept which includes two QC active sections which generate slightly offset wavelengths of IR radiation in a material which is also highly optically nonlinear and may generate a THz signal via DFG within the QCL itself [59]. There are still some considerable technical challenges to be overcome in such devices, and indeed as with any $\mathrm{THz}$ QCL the efficiency decreases with increasing operating temperature, giving up to $7 \mu \mathrm{W}$ $\mathrm{THz}$ output power at liquid nitrogen temperatures and around $300 \mathrm{nW}$ output at room temperature. Incidentally, tunability between around $4.5 \mathrm{THz}$ and $4.8 \mathrm{THz}$ is also observed, as the emission peak from each integrated QC IR source tends to drift slightly with operating temperature. In these devices, the limiting factor appears to be the decreasing efficiency of the QC IR lasing modules with temperature, rather than the nonlinear optical conversion to $\mathrm{THz}$ radiation. 


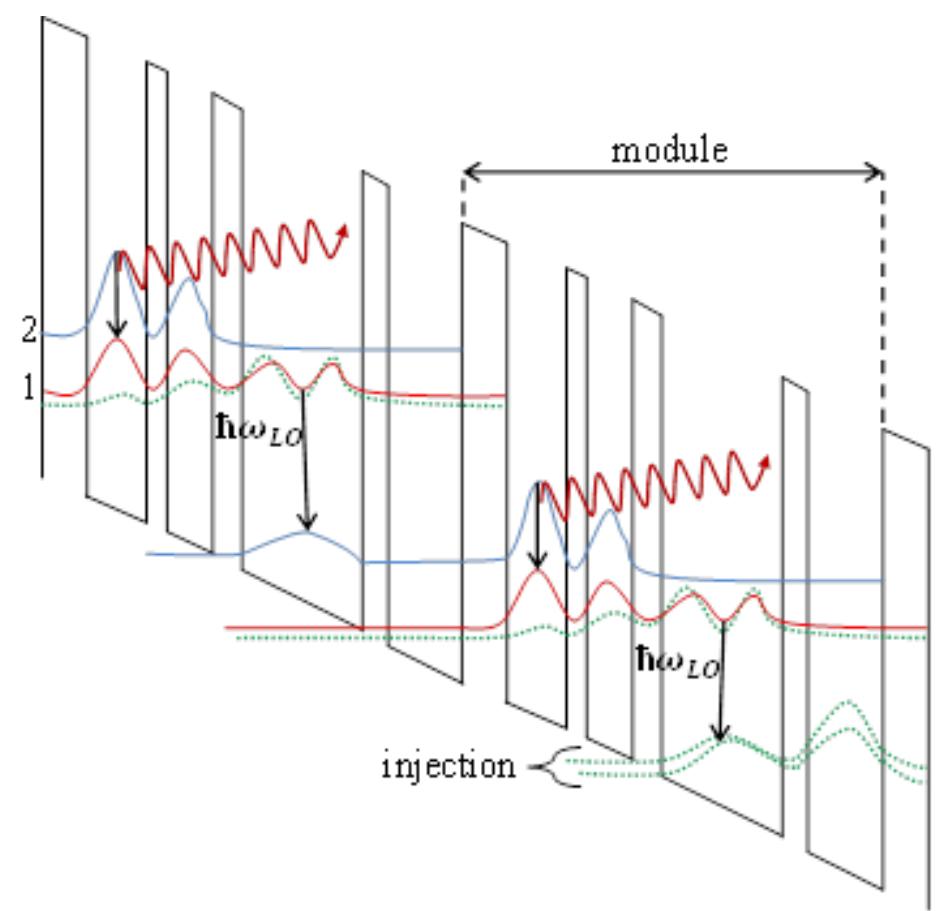

Fig. 2. Example energy band diagram of electron-tunnelling throughout successive QW heterostructure modules within a resonant-phonon QCL structure. Lasing levels 1 and 2 are indicated, as well as radiative and phonon-coupled charge carrier transitions.

Stefano Barbieri of TeraView Limited in 2005 patented a so-called bound-to-continuum [66] THz QCL device in which the upper lasing state of each active module extends significantly through the injection barrier into the next module [67]. This is in order to improve current flow and current density threshold whilst enhancing gain in the device by maintaining a high population density in the upper state and improving the coupling of the upper lasing level with the other states in the successive lasing module. This allows the QCL to generate a $3 \mathrm{THz}$ pulsed signal up to around $95 \mathrm{~K}$, and a $\mathrm{CW}$ signal up to $70 \mathrm{~K}$.

Lee and Wanke of Sandia Corporation in 2009 patented an integrated QCL and Schottky diode transceiver system which uses an intermediate-frequency mixing technique between the $\mathrm{THz}$ field detected by antenna connected to the Schottky diode, and the local oscillator (LO) signal (around 2.92THz, for example) generated by the on-board QCL [68], as illustrated in Figure 3. The nature of the $\mathrm{THz}$ signal is analysed by sweeping the on-board spectrum analyser. This system allows a reliable, portable remote spectral analyser/imager/sensor to be made, which can analyse $\mathrm{THz}$ spectral properties within a limited range of the LO frequency. The details of the invention include a note that the device meets important requirements such as a "high operating temperature", and that this temperature may be increased further when operating in 'passive' mode using only the receiver antenna, but no specific operating temperature range is given and liquid-nitrogen-range operating temperatures may be assumed. 
The operating temperature of THz-capable QCL's is slowly approaching the working range of thermoelectric coolers, however, and in particular the method of dual IR-signal generation and DFG within the QCL medium looks as though it may be able to provide practical operating efficiency in the near future.

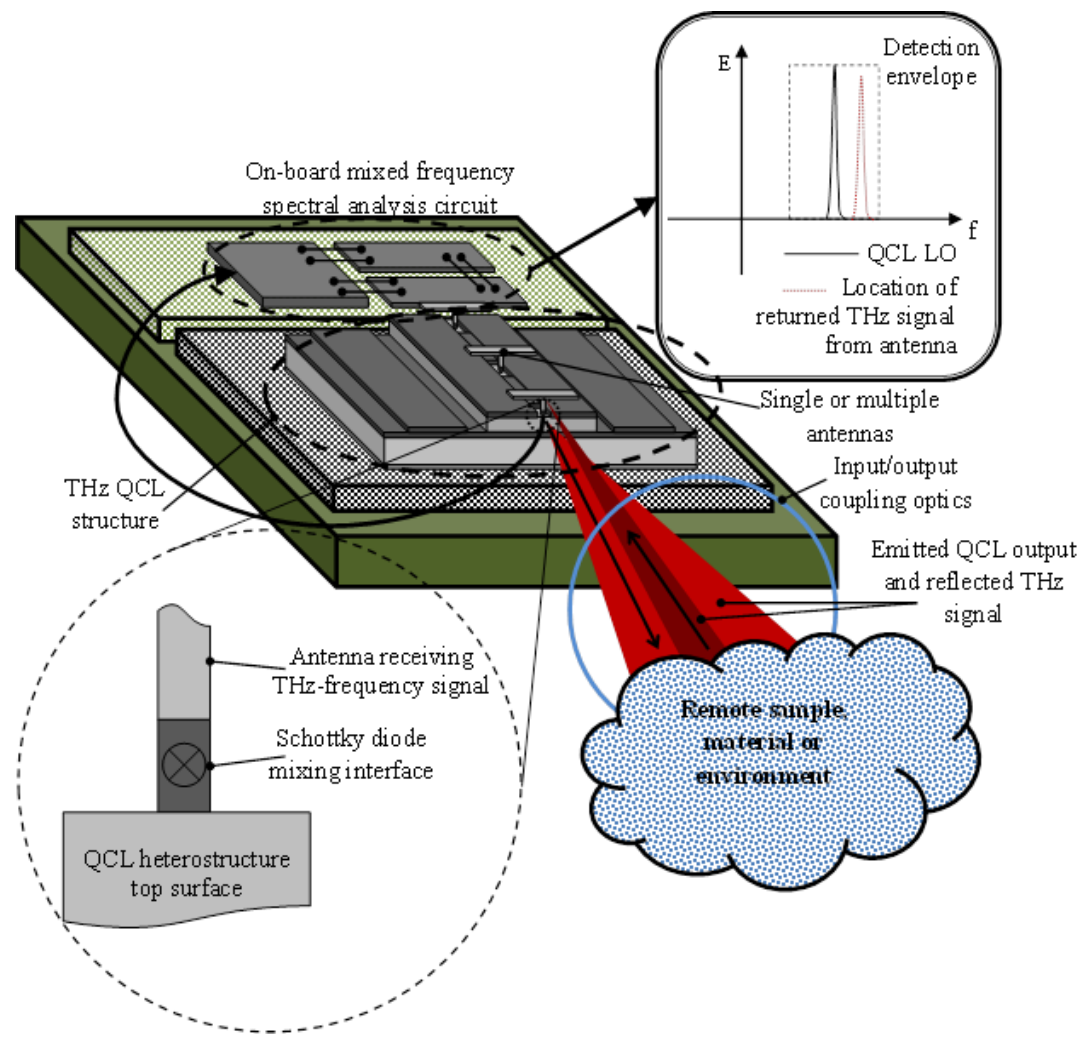

Fig. 3. Exemplary schematic layout of the integrated THz transceiver by Lee \& Wanke (not to scale). The waveform within the semiconductor gain medium of the QCL is at a maximum near the semiconductor-metal surface. This allows the device to take some fraction of the QCL's (THz) signal power for use in mixing with the incoming THz signal picked up by the integrated antenna(s).

\section{High Frequency Diodes and Photodiodes}

As optical systems gradually approach operational frequencies in the THz range from 'above' the THz gap, electronic devices are gradually approaching the gap from frequencies 'below' it. Research into the improved (higher frequency) bandwidth of structures and materials used in photodiodes (PD's) and switches is ongoing and several promising full solutions have emerged, most notably including: the $\mathrm{THz}$ resonant tunnelling diode (RTD); and the uni-travellingcarrier-photodiode (UTC-PD). Both concepts are based upon the fast transport of electrons through semiconductor layers or junctions, but differ fundamentally in the harnessing of this ultrafast switching for the purpose of $\mathrm{THz}$ output signal generation.

The RTD $[69,70]$ is based on the confinement of electrons within a quantum well (e.g. undoped $4.5 \mathrm{~nm}$ of $\operatorname{In}_{0.8} \mathrm{Ga}_{0.2} \mathrm{As}$ ) and the subsequent tunnelling through the confining 
heterobarriers (i.e. each $1.4 \mathrm{~nm}$ of AlAs) upon application of an appropriate external bias, which results in a region of negative differential conductance (NDC) in the device's I-V response curve. A tunnel diode oscillator utilises this region of NDC, which is inherent in the resultant electron-hole band edge mismatching properties over the heterobarrier structure, to modulate the applied bias into an oscillating signal when coupled with a parallel inductor-capacitor-resistor circuit (Figure 4). This application of the tunnel diode is now mature, but engineering of the device structure and materials to achieve $\mathrm{THz}$-scale frequencies is still a matter of ongoing research.

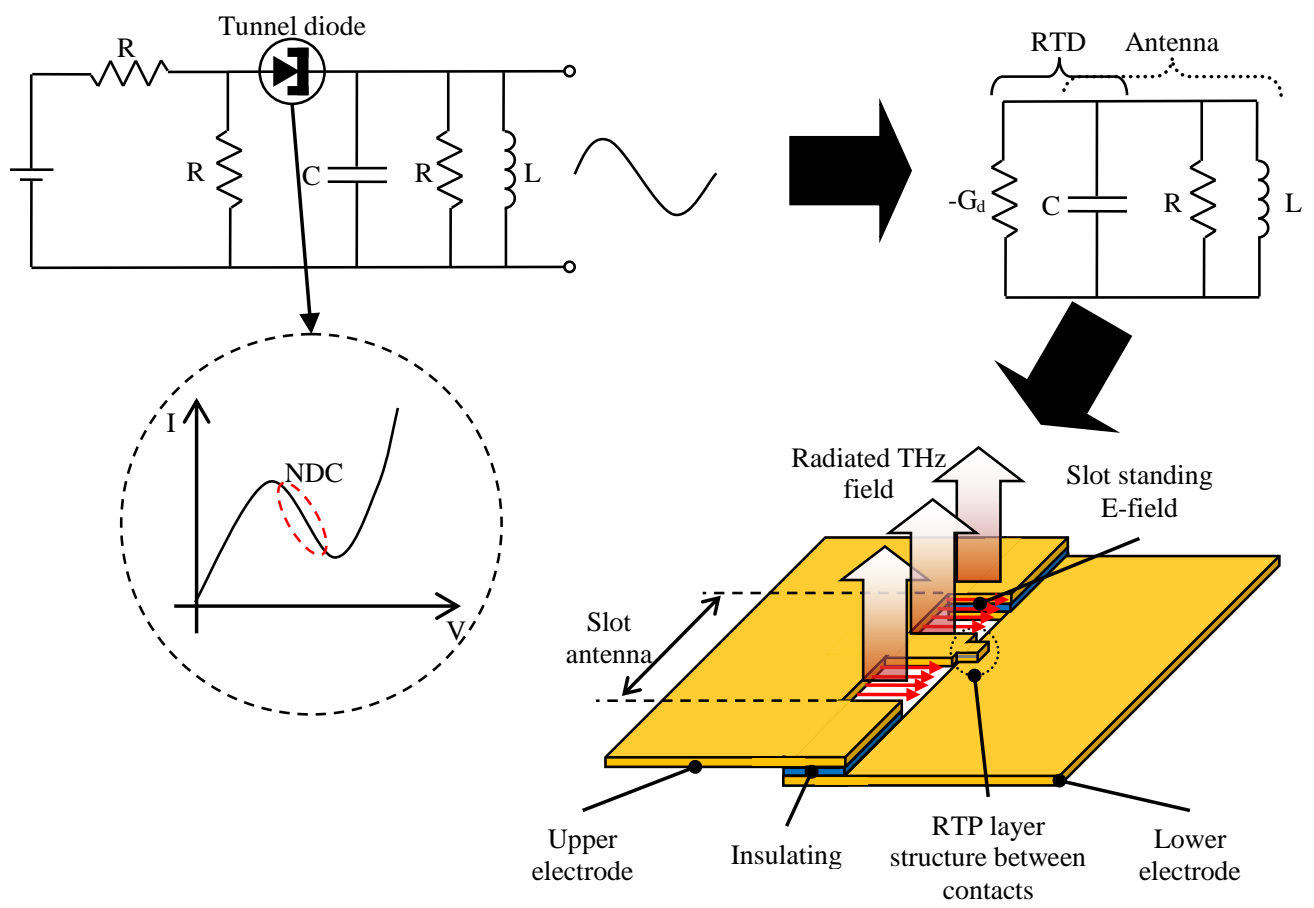

Fig. 4. Basic principles of the RTD THz signal source. Top left: circuit diagram of the fundamental RTP electrical operation and I-V curve. The bias point of the diode is within the NDC region. Top right: equivalent circuit of the RTD and integrated slot antenna. Oscillation is achieved when the NDC absolute value is greater than the antenna radiation losses. Bottom right: simplified structural layout of the integrated device. The two $\mathrm{Au} / \mathrm{Pd} / \mathrm{Ti}$ contacts are bridged by the RTD heterostructure, such that electrical oscillation between them is at the fundamental and higher harmonics of the RTD heterobarrier quantum constraints, and the resulting standing field between them is radiated by the slot antenna.

Masahiro Asada and Safumi Suzuki of the Tokyo Institute of Technology are the main modern research proponents of the technology as applied to the $\mathrm{THz}$ field, and along with Sugiyama Hiroki and Yokoyama Haruki of the Nippon Telegraph \& Telephone Corp. have patented an optimised RTD materials structure and device design [71] such as the one illustrated in Figure 4, and includes their recent work on "spiked doping" structures. Optimisations in material doping, resonant tunnelling and transit lifetime reductions and device layout has allowed the group to achieve an impressive oscillation frequency just above $1 \mathrm{THz}$ at room 
temperature, with a $\mathrm{THz}$ output power of $7 \mu \mathrm{W}$ and a tunability of around $40 \mathrm{GHz}$ which is achieved by altering the RTD bias [72]. This makes the RTD a serious competitor in the fields of both compact $\mathrm{THz}$ radiation sources and ultrafast electronic devices.

UTC-PDs may be thought of as the 'next generation' of photodiodes, which operate using the same principles but with an optimised carrier transport mechanism in comparison with their traditional p-i-n formats. P-i-n photodiodes operate using the band-structure principle of electron-hole recombination upon photonic stimulation of the active area. In this process, the rate of recombination and hence the operational frequency of such a device is limited mainly by the transport time of holes in the active region, and so Ishibashi et al. investigated the operation of a PD which utilises only fast electron transport in the switching process [41].

In this device, the active layer is characterised by a p-type light absorption layer and a depleted carrier collection layer. Electrons travel only towards the collection layer, the bandgap of which is graded via doping and transport is extremely fast (electron velocity overshoot in the collection layer may be $\sim 4 \times 10^{7} \mathrm{~cm} / \mathrm{s}$, as opposed to hole velocity $\sim 5 \times 10^{6} \mathrm{~cm} / \mathrm{s}$ ). Since the majority carrier in this section is holes, transport is determined by the electron current and the UTC-PD is able to operate (switch) over much shorter timescales and hence at much higher frequencies, which is now within the THz region. Ito et al. reported operation of a InP/InGaAs UTC-PD with a $3 \mathrm{~dB}$ bandwidth of $310 \mathrm{GHz}$ and a FWHM pulse width of $0.97 \mathrm{ps}$ [73], which operated at the technologically significant telecommunications wavelength of $1.55 \mu \mathrm{m}$. This technology is most exemplary because it has been developed to marry the challenges of sub-ps semiconductor switch materials with $\mathrm{THz}$ optoelectronics, reported in an interesting paper by Ito et al. in 2005 [74]. In this development, the ultrafast switching capability of the UTC-PD at long IR wavelengths is implemented in a $\mathrm{THz}$ heterodyne receiver structure and was reported to generate a $1.04 \mathrm{THz}$ output power of $2.3 \mu \mathrm{W}$ at room temperature and may be operated up to $1.5 \mathrm{THz}$, the details of which are discussed further in the next section.

\section{THz Heterodyne Sources}

Heterodyning or "mixing" techniques are now mature, and the method of superimposing two oscillating signals in a mixer device for analysis of a down-converted resultant signal has been applied for the purpose of THz DFG in devices based on sub-ps photoconductive (PC) materials for nearly two decades now [45, 47, 48, 75]. Instead of mixing incoming radiation with a local oscillator source, the active region in a $\mathrm{THz}$ heterodyne receiver is based on a material with high absorbance in the IR region and high EO susceptibility, on which two IR optical pump beams are incident, and are mixed. Several different conditions are to be met in the design of the device if efficient $\mathrm{THz}$ generation is to be realised, categorised by three main system stages: (1) the optical pump(s), (2) the photomixer device and materials and (3) the $\mathrm{THz}$ outcoupling/optics. 


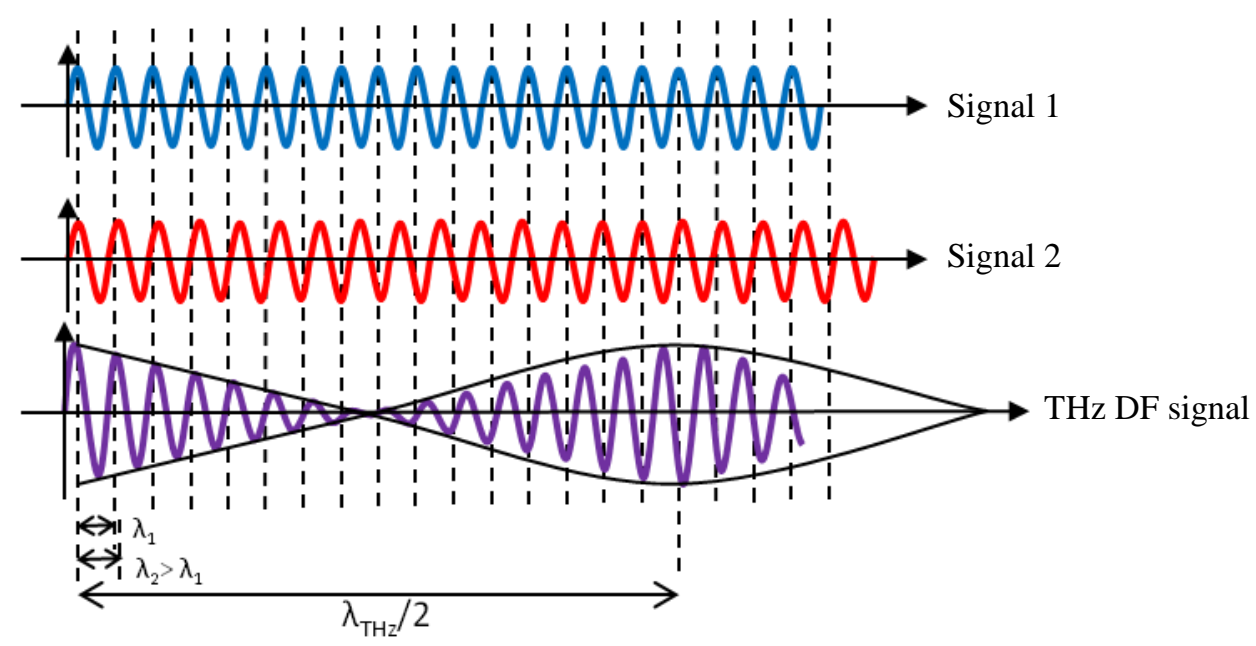

Fig. 5. Sketch of the concept of $\mathrm{THz}$ difference frequency generation (DFG) via beating or mixing of two IR pump signals.

\section{Optical Pumping of THz Heterodyne Receiver}

The pump source for the device will typically comprise of two narrow-linewidth IR signals of wavelengths somewhere between $800 \mathrm{~nm}$ and $1550 \mathrm{~nm}$ from either pulsed or CW coherent sources. The pump signals with both have an energy above the bandgap of the photomixer active material, and their longitudinal wavelengths should be offset by say a few $\mathrm{nm}$, depending on the spectral range they are at, whilst maintaining a suitable spatial overlap particularly as they are incident on the photomixer active layer. The waves are 'beat' together in the active EO material to give a resultant beat frequency in the $\mathrm{THz}$ range (Figure 5).

The modulation of electronic polarity within the active region will switch it electronically essentially between a conductive and non-conductive state, and this $\mathrm{THz}$ switching signal may be loaded by, for example, a dipole antenna which is integrated with the PC layer which can re-radiate this signal through the substrate into free space. Several different pump methods and devices are suitable for this technique - the seminal $\mathrm{THz}$ optoelectronics research was conducted using femtosecond (fs) pulses from a Ti:Sapphire laser which switches the device not by DFG but by generating a photocurrent over the fs timescale, which allowed the device to re-emit a corresponding EM pulse in the temporal domain, the Fourier Transform of which was a broadband $\mathrm{THz}$ pulse [76-78]. This method allows the generation of relatively high-power broadband $\mathrm{THz}$ output signals above $100 \mu \mathrm{W}$ with an optical pump power of several hundred milliwatts, and the bandwidth is determined by the pulsewidth of the pump signal. This output power is 2 orders of magnitude greater than that expected from current $\mathrm{CW}$ heterodyne systems, but is limited by the need for powerful and bulky ultrafast laser drivers and the control of $\mathrm{THz}$ bandwidth is rudimentarily based upon tuning the pulse width of said driver(s). An 
excellent comparison of state-of-the-art pulsed "photoconductive switches" and CW heterodyne operating conditions was published by Brown earlier this year [79].

The generation of so-called "dual-modal" THz photomixer optical pump signals has been accomplished in several different ways. This includes: systems of tunable multiterahertz difference frequency generation via two-wavelength laser diode (LD) arrays [80]; spatial combination of two pulsed $\mathrm{Ti}_{2} \mathrm{Al}_{2} \mathrm{O}_{3}$ lasers or CW LD's [48]; single monolithic duallongitudinal-mode LD's [81]; spectral tuning of a single LD using gratings and an etalon [82]; and dual-mode tuning of a single LD using single [83] and/or multiple ("multiplexed") volume Bragg gratings (VBG's) in an external cavity [84], a typical output spectrum of which is given in Figure 6.

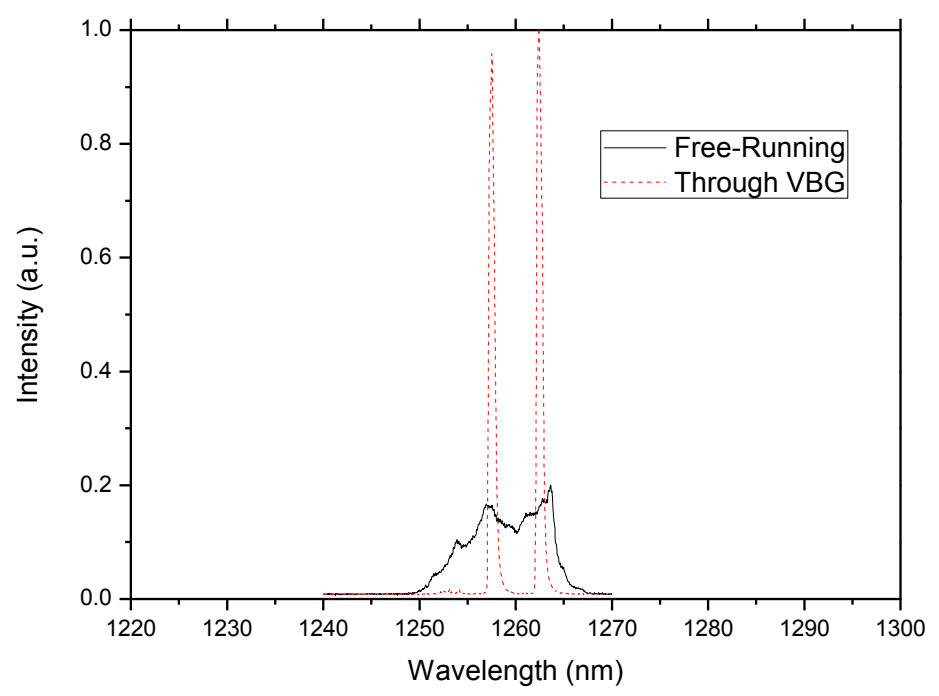

Fig. 6. Example of stable dual-modal spectral output achieved by using a multiplexed VBG in an external cavity feedback setup with a single quantum-dot diode laser.

\section{The Photomixer Device Including the 'Photoconductive Antenna'}

The design of the photomixer device itself is more complex, and a great deal of work is going into a wide range of optimisations with the aim of improving the optical-THz efficiency of such devices. At the heart of this research is still the issue of the growth conditions of sub-ps materials, and work is still ongoing to develop and improve material(s) with optimal characteristics such as high dark-resistivity, high charge carrier mobility as well as ultra-short carrier lifetimes to allow the required sub-ps response whilst retaining high internal gain. This work is essentially the basis of all semiconductor $\mathrm{THz}$ devices and stemmed from experiments based on the engineering of defects within the semiconductor medium, such as radiationdamaged silicon-on-sapphire $[14,16]$ and GaAs grown at low-temperatures (LT-GaAs) by molecular beam epitaxy (MBE) with an arsenic overpressure $[85,86]$ which encourages As 
interstitial defects to be deposited at high densities throughout the crystal. These defects in each case act as trapping sites and effectively shorten the (photo)carrier lifetimes within these materials down to sub-ps ranges whilst still retaining relatively high carrier mobility [87, 88]. $\mathrm{THz}$ photomixer materials are now generally based on modification of GaAs due to its high absorption of IR radiation, and $\mathrm{THz}$ radiation has been generated from materials such as GaAs with incorporated layers of self-assembled quantum-dot (QD) nanoislands [89] and narrower bandgap materials such as LT-GaBiAs [90], InGaAs [91], LT-InGaAs [92-94], InGaAs with incorporated QD's [52, 95, 96] and InGaAs with dopants such as Fe [97]. The inclusion of In within the MBE blend allows the material to operate with optical pumps with up to telecomsrange wavelengths, whilst still retaining relatively high gain, and the inclusion of QD gain layers allows an extra degree of wavelength, gain and carrier trapping time tunability.

These materials all operate as effective $\mathrm{THz}$ photomixers at room temperature and make for very small devices, but are limited by such crucial factors such as thermal breakdown [98] under modest CW pump power (above a few tens of $\mathrm{mW}$, say, depending upon the thermal conductivity of the blend/structure), and quantum efficiency roll-off as the operating frequency increases above $1 \mathrm{THz}$ due to carrier lifetime and RC-time factor constraints. Thermal conductivity of the active layer may be improved by inclusion of $\mathrm{Al}$ within the blend or multilayered structure [99], for example. Heatsinking is improved by running the device at liquid nitrogen temperatures also, which allows the device to be pumped at higher power and thus to achieve higher THz output power [98], but this of course is typically not ideal for the intended application(s). The quantum efficiency of the active region may be improved in many different ways, most notably by strengthening and further homogenising the electric-field throughout the depth of the active layer(s) using electrodes deposited over the entire active region depth, rather
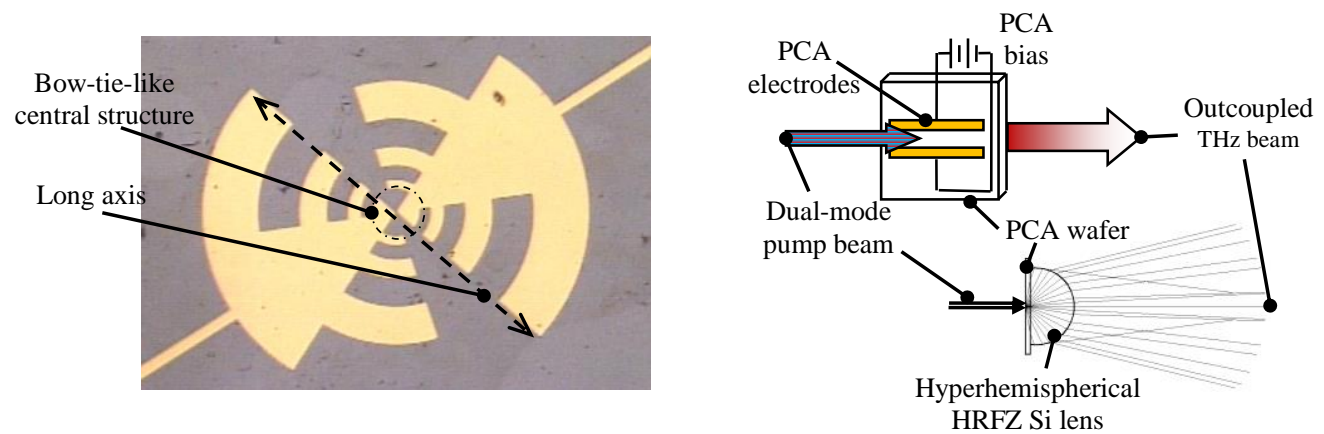

Fig. 7. Left: Example of a Au/Ti broadband THz antenna deposited over a LT-GaAs substrate. The central structure within which the pump beam is incident behaves as a bow-tie emitter with a resonant frequency around $350 \mathrm{GHz}$. The long axis indicated is $1280 \mu \mathrm{m}$ in length, comprising the two graded tooth emitter sections for a variance of emission wavelengths. Right: basic layout of the photomixer setup. The dual-mode pump beam is incident on the PCA gap, and the THz frequency photocurrent is re-radiated by the integrated PCA through the substrate, which is further collimated using a HRFZ Si lens $\left(n_{G a A s} \approx 3.6, n_{S i} \approx 3.42\right)$. 
than just on the surface. Roehle et al. achieved enhanced THz efficiency of photomixer devices etched in a mesa-structure with contacts laid over various active layer depths [54], for example. Other attempts have been made to confine the optically active photomixer region to the layer surface where the E-field is strongest by using a buried distributed Bragg reflector (DBR) underneath the active layer [51,99-101] to reflect the pump signal back and forth between the layer surface and the DBR and increase the effective absorption length, but the thermal constraints of the material(s) still apply and output powers are not particularly enhanced any more than with the inclusion of the thermal-conditioning Al-based layers.

Optimisation of the photomixer's internal E-field characteristics leads into the investigation of antenna electrode structuring. Not only is the distribution of field strength within the active layer important, but in $\mathrm{CW}$ PC THz antennas in particular, the layout of the antenna electrode layer/structure itself (herein referred to as the 'antenna') is crucial to its optoelectronic performance and the nature of the THz output signal. Early PC THz antennas were essentially micron-scale Hertzian dipole antennas, which were held at an appropriate electrical bias and were current-loaded when the active layer in the area between dipole gap was optically pumped/gated by the fs-pulse or $\mathrm{CW}$ beat signal. This gave limited performance in the $\mathrm{CW}$ regime and since then, enhanced $\mathrm{THz}$ output has been investigated with the use of broadband-emission, higher power antennas such as self-complimentary spiral $[49,98]$ and circularly-toothed log-periodic designs [53, 102, 103], as well as traditional 'bow-tie' designs. An example of an $\mathrm{Au} / \mathrm{Ti} \log$-periodic $\mathrm{PC} \mathrm{THz}$ antenna is given in Figure 7. Note that the previously mentioned work in which a UTC-PD was integrated with a CW THz PC antenna utilised this antenna structure with the UTC-PD at the centre [74].

Antenna structures like these allow the generation of broadband $\mathrm{THz}$ radiation (to beyond $1 \mathrm{THz}$, but with a signal fall-off due to carrier and RC lifetime constraints as mentioned previously) at relatively high powers (typically up to around a few microns). The use of resonant dipole and dual-dipole structures allows higher powers to be generated around the specific respective resonant frequencies up to and beyond $2 \mathrm{THz}$ [104] but this involves careful design and production of more detailed, "interdigitated" electrode fingers which span the PC antenna gap, as well as inductive 'choke' structures separated by a quarter resonant wavelength along the length of the stripline leading to the dipole(s). The inductive choke features help balance the capacitive effects at the design frequency which otherwise would allow generated $\mathrm{THz}$ signals to leak down the bias lines. The interdigitated fingers are normally etched by electron beam lithography, as the finger and inter-finger gap width are generally sub-micron. The reason for this is manifold: to enhance the quantum gain of generated photocurrent and improve coupling to the antenna for re-radiation - photocarriers must travel through the highly inter-atomically inconsistent crystal with very short lifetimes; thinner fingers reduce the amount of pump signal which is blocked over the active area; and capacitive effects are reduced if 
smaller electrodes are used while working at such a short separation. Interdigitated finger PC antenna gaps are also implemented at the heart of $\mathrm{BB}$ emission structures for improved gain [53]. CW PC THz antennas exhibit output powers which are characteristically quadratic with increasing optical pump power and electrode bias, as is also the case with pulsed-mode PC antennas.

\section{THz Outcoupling and Optics}

It is observed that $\mathrm{THz}$ radiation generated in such photomixer devices exhibits a very high divergence angle relative to the optic axis, and this is typically remedied with the use of a hyperhemispherical high resistivity float zone silicon (HRFZ-Si) lens abutted to the rear of the antenna chip. The divergent $\mathrm{THz}$ beam is emitted from the active photomixer volume, through the substrate (the substrate has a refractive index at $\mathrm{THz}$ wavelengths typically around 3.4 for GaAs, which the generated THz beam is simply geometrically 'captured by' much easier than air when at the GaAs-air interface before propagating) and is focussed to some degree by the $\mathrm{Si}$ lens, after which point it may be refocused and/or collimated using parabolic mirrors. The $\mathrm{THz}$ beams may also be analysed using optics in configurations such as FIR/THz interferometers [105] and grid polarisers and various low-loss THz lenses are now commercially commonplace $[106,107]$.

$\mathrm{CW} \mathrm{THz}$ heterodyne sources are not yet quite commercially commonplace due to their low $\mathrm{THz}$ output powers, but are certainly in use in research applications. An entire system of this type constitutes a very compact and relatively efficient, frequency tunable room temperature source which may be constructed very economically and is relatively robust and low-power. As the work continues into thermal conditioning, optimal antenna design and optoelectronic gain, these sources are sure to become even more widespread in the $\mathrm{THz}$ signalling field in the near future.

\section{CURRENT \& FUTURE DEVELOPMENTS}

$\mathrm{THz}$ (opto)electronics is a rapidly expanding field, as improvements in the technology needed for the implementation of the wide range of valuable $\mathrm{THz}$ analytical applications is now gradually being realised. High-power, pulsed $\mathrm{THz}$ radiation sources and detectors are now relatively commonplace in research and industry as well as commercial products for imaging applications, for example, but the limitations of these devices such as frequency domain tunability and selectivity (pulsed sources are inherently broadband), optoelectronic efficiency and general practicality (e.g. size, mechanical stability, power consumption) mean that there is still vast scope for development particularly in the $\mathrm{CW}$, frequency-domain arena. To this end, several successful methods are already employed and development is ongoing, primarily in the material engineering of: ultrafast semiconductors for improved charge carrier control and $\mathrm{THz}$ 
frequency operation; and nonlinear EO crystals for improved second-order frequency conversion and waveguiding. Presented in this review is a very general overview in current competing compact room-temperature $\mathrm{THz}$ sources and some of the noteworthy recent patents resulting, and we note that researchers are still pursuing a highly efficient source of this type which may be used eventually as practical, low-power, robust optoelectronic devices over a range of research activities and disciplines, as well as commercial, military and industrial applications which currently are managed by large, inefficient and complex machinery such as X-Ray devices.

\section{ACKNOWLEDGEMENTS}

The authors would like to thank the EPSRC for support under grant EP/H015795/1

\section{CONFLICT OF INTEREST}

The authors declare no conflict of interest.

\section{REFERENCES}

[1] J. F. Federici, B. Schulkin, F. Huang, D. Gary, R. Barat, F. Oliveira, and D. Zimdars, "THz imaging and sensing for security applications - explosives, weapons and drugs," Semiconductor Science and Technology, vol. 20, pp. S266-S280, 2005.

[2] H. Quast and T. Loffler, "Towards Real-Time Active THz Range Imaging for Security Applications," ICEAA: 2009 International Conference on Electromagnetics in Advanced Applications, Vols 1 and 2, pp. 501-504, 2009.

[3] A. G. Davies, A. D. Burnett, W. Fan, E. H. Linfield, and J. E. Cunningham, "Terahertz spectroscopy of explosives and drugs," Materials Today, vol. 11, pp. 18-26, 2008.

[4] H. B. Liu, Y. Q. Chen, and X. C. Zhang, "Characterization of anhydrous and hydrated pharmaceutical materials with $\mathrm{THz}$ time-domain spectroscopy," Journal of Pharmaceutical Sciences, vol. 96, pp. 927-934, 2007.

[5] M. R. Scarfi, M. Romano, R. Di Pietro, O. Zeni, A. Doria, G. P. Gallerano, E. Giovenale, G. Messina, A. Lai, G. Campurra, D. Coniglio, and M. D'Arienzo, "THz exposure of whole blood for the study of biological effects on human lymphocytes," Journal of Biological Physics, vol. 29, pp. 171-177, 2003.

[6] K. J. Siebert, T. Loffler, H. Quast, M. Thomson, T. Bauer, R. Leonhardt, S. Czasch, and H. G. Roskos, "All-optoelectronic continuous wave $\mathrm{THz}$ imaging for biomedical applications," Physics in Medicine and Biology, vol. 47, pp. 3743-3748, 2002.

[7] P. H. Siegel, "Terahertz Technology," IEEE Transactions on Microwave Theory and Techniques, vol. 50, pp. 910-928, 2002.

[8] T. Yajima and N. Takeuchi, "Far-Infrared Difference-Frequency Generation by Picosecond Laser pulses," Japanese Journal of Applied Physics, vol. 9, p. 1361, 1970.

[9] K. H. Yang, P. L. Richards, and Y. R. Shen, "Generation of Far-Infrared Radiaton by Picosecond Light Pulses in $\mathrm{LiNbO}_{3}, "$ Applied Physics Letters, vol. 19, p. 320, 1971.

[10] D. H. Auston, K. P. Cheung, J. A. Valdmanis, and D. A. Kleinman, "Cherenkov Radiation from Femtosecond Optical Pulses in Electro-Optic Media," Physical Review Letters, vol. 53, pp. 1555-1558, 1984.

[11] D. H. Auston and M. C. Nuss, "Electrooptic Generation and Detection of Femtosecond Electrical Transients," IEEE Journal of Quantum Electronics, vol. 24, pp. 184-197, 1988. 
[12] D. H. Auston, "Impulse-Response of Photoconductors in Transmission-Lines," IEEE Journal of Quantum Electronics, vol. 19, pp. 639-648, 1983.

[13] P. R. Smith, D. H. Auston, and W. M. Augustyniak, "Measurement of GaAs FieldEffect Transistor Electronic Impulse-Response by Picosecond Optical Electronics," Applied Physics Letters, vol. 39, pp. 739-741, 1981.

[14] D. H. Auston, "Picosecond Optoelectronic Switching and Gating in Silicon," Applied Physics Letters, vol. 26, pp. 101-103, 1975.

[15] D. H. Auston, A. M. Johnson, P. R. Smith, and J. C. Bean, "Picosecond Optoelectronic Detection, Sampling, and Correlation-Measurements in Amorphous-Semiconductors," Applied Physics Letters, vol. 37, pp. 371-373, 1980.

[16] P. R. Smith, D. H. Auston, A. M. Johnson, and W. M. Augustyniak, "Picosecond Photoconductivity in Radiation-Damaged Silicon-on-Sapphire Films," Applied Physics Letters, vol. 38, pp. 47-50, 1981.

[17] L. Rui, C. M. Gu, L. R. He, W. Sen, W. Z. Shen, O. Hiroshi, and Q. X. Guo, "Generation of terahertz radiation via optical rectification and electro-optic detection in ZnTe crystals," Acta Physica Sinica, vol. 53, pp. 1217-1222, 2004.

[18] Q. Chen, M. Tani, Z. P. Jiang, and X. C. Zhang, "Electro-optic transceivers for terahertz-wave applications," Journal of the Optical Society of America B-Optical Physics, vol. 18, pp. 823-831, 2001.

[19] Y. S. Lee, "Generation of narrow-band terahertz radiation via optical rectification of femtosecond pulses in periodically poled lithium niobate," Applied Physics Letters, vol. 76, p. 2505, 2000.

[20] K. Kawase, M. Sato, T. Taniuchi, and H. Ito, "Coherent tunable THz-wave generation from LiNbO3 with monolithic grating coupler," Applied Physics Letters, vol. 68, pp. 2483-2485, 1996.

[21] D. J. Cook and R. M. Hochstrasser, "Intense terahertz pulses by four-wave rectification in air," Optics Letters, vol. 25, pp. 1210-1212, Aug 152000.

[22] H. Hamster, A. Sullivan, S. Gordon, W. White, and R. W. Falcone, "Subpicosecond, Electromagnetic Pulses from Intense Laser-Plasma Interaction," Physical Review Letters, vol. 71, pp. 2725-2728, Oct 251993.

[23] T. Loffler and H. G. Roskos, "Gas-pressure dependence of terahertz-pulse generation in a laser-generated nitrogen plasma," Journal of Applied Physics, vol. 91, pp. 2611-2614, Mar 12002.

[24] M. Kress, T. Loffler, S. Eden, M. Thomson, and H. G. Roskos, "Terahertz-pulse generation by photoionization of air with laser pulses composed of both fundamental and second-harmonic waves," Optics Letters, vol. 29, pp. 1120-1122, May 152004.

[25] T. Bartel, P. Gaal, K. Reimann, M. Woerner, and T. Elsaesser, "Generation of singlecycle THz transients with high electric-field amplitudes," Optics Letters, vol. 30, pp. 2805-2807, Oct 152005.

[26] K. Y. Kim, J. H. Glownia, A. J. Taylor, and G. Rodriguez, "Terahertz emission from ultrafast ionizing air in symmetry-broken laser fields," Optics Express, vol. 15, pp. 4577-4584, Apr 162007.

[27] X. Xie, J. M. Dai, and X. C. Zhang, "Coherent control of THz wave generation in ambient air," Physical Review Letters, vol. 96, Feb 242006.

[28] C. D'Amico, A. Houard, M. Franco, B. Prade, A. Mysyrowicz, A. Couairon, and V. T. Tikhonchuk, "Conical forward $\mathrm{THz}$ emission from femtosecond-laser-beam filamentation in air," Physical Review Letters, vol. 98, p. 235002, 2007-Jun-8 2007.

[29] J. Faist, F. Capasso, D. L. Sivco, C. Sirtori, A. L. Hutchinson, and A. Y. Cho, "Quantum Cascade Laser," Science, vol. 264, pp. 553-556, 1994.

[30] M. Beck, D. Hofstetter, T. Aellen, J. Faist, U. Oesterle, M. Ilegems, E. Gini, and H. Melchior, "Continuous wave operation of a mid-infrared semiconductor laser at room temperature," Science, vol. 295, pp. 301-305, 2002.

[31] R. Kohler, A. Tredicucci, F. Beltram, H. E. Beere, E. H. Linfield, A. G. Davies, D. A. Ritchie, R. C. Iotti, and F. Rossi, "Terahertz semiconductor-heterostructure laser," Nature, vol. 417, pp. 156-159, 2002. 
[32] G. Scalari, S. Blaser, J. Faist, H. Beere, E. Linfield, D. Ritchie, and G. Davies, "Terahertz emission from quantum cascade lasers in the quantum Hall regime: Evidence for many body resonances and localization effects," Physical Review Letters, vol. 93, 2004.

[33] M. Troccoli, S. Corzine, D. Bour, J. Zhu, O. Assayag, L. Diehl, B. G. Lee, G. Hofler, and E. Capasso, "Room temperature continuous-wave operation of quantum-cascade lasers grown by metal organic vapour phase epitaxy," Electronics Letters, vol. 41, pp. 1059-1060, 2005.

[34] B. S. Williams, S. Kumar, Q. Hu, and J. L. Reno, "Operation of terahertz quantumcascade lasers at $164 \mathrm{~K}$ in pulsed mode and at $117 \mathrm{~K}$ in continuous-wave mode," Optics Express, vol. 13, pp. 3331-3339, 2005.

[35] B. S. Williams, "High-power terahertz quantum-cascade lasers," Electronics Letters, vol. 42, p. 89, 2006.

[36] B. S. Williams, "Terahertz quantum-cascade lasers," Nature Photonics, vol. 1, pp. 517525, 2007.

[37] M. A. Belkin, Q. J. Wang, C. Pflugl, A. Belyanin, S. P. Khanna, A. G. Davies, E. H. Linfield, and F. Capasso, "High-Temperature Operation of Terahertz Quantum Cascade Laser Sources," IEEE Journal of Selected Topics in Quantum Electronics, vol. 15, pp. 952-967, 2009.

[38] T. Ishibashi, T. Furuta, H. Fushimi, and H. Ito, "Photoresponse characteristics of unitraveling-carrier photodiodes," San Jose, CA, USA, 2001, pp. 469-479.

[39] T. Ishibashi, N. Shimizu, S. Kodama, H. Ito, T. Nagatsuma, and T. Furuta, "Unitraveling-carrier photodiodes," in Tech. Dig. Ultrafast Electronics and Optoelectronics, Lake Tahoe, 1997, pp. 83-87.

[40] T. Ohno, S. Fukushima, Y. Doi, Y. Muramoto, and Y. Matsuoka, "Application of unitraveling-carrier waveguide photodiodes in base stations of a millimeter-wave fiberradio system," presented at the Int. Topical Meeting Microwave Photonics, Melbourne, Australia, 1999.

[41] T. Ishibashi, T. Furuta, H. Fushimi, S. Kodama, H. Ito, T. Nagatsuma, N. Shimizu, and Y. Miyamoto, "InP/InGaAs uni-traveling-carrier photodiodes," IEICE Transactions on Electronics, vol. E83C, pp. 938-949, 2000.

[42] H. Ito, S. Kodama, Y. Muramoto, T. Furuta, T. Nagatsuma, and T. Ishibashi, "Highspeed and high-output InP-InGaAs unitraveling-carrier photodiodes," IEEE Journal of Selected Topics in Quantum Electronics, vol. 10, pp. 709-727, 2004.

[43] S. Matsuura and H. Ito, "Generation of CW terahertz radiation with photomixing," Terahertz Optoelectronics, vol. 97, pp. 157-202, 2005.

[44] M. Tani, P. Gu, M. Hyodo, K. Sakai, and T. Hidaka, "Generation of coherent terahertz radiation by photomixing of dual-mode lasers," Optical and Quantum Electronics, vol. 32, pp. 503-520, 2000.

[45] E. R. Brown, F. W. Smith, and K. A. McIntosh, "Coherent Millimeter-Wave Generation by Heterodyne Conversion in Low-Temperature-Grown Gaas Photoconductors," Journal of Applied Physics, vol. 73, pp. 1480-1484, 1993.

[46] E. R. Brown, K. A. McIntosh, F. W. Smith, K. B. Nichols, M. J. Manfra, C. L. Dennis, and J. P. Mattia, "Milliwatt Output Levels and Superquadratic Bias Dependence in a Low-Temperature-Grown GaAs Photomixer," Applied Physics Letters, vol. 64, pp. 3311-3313, 1994.

[47] E. R. Brown, K. A. McIntosh, K. B. Nichols, and C. L. Dennis, "Phohtomixing up to 3.8-THz in Low-Temperature-Grown GaAs," Applied Physics Letters, vol. 66, pp. 285287, 1995.

[48] K. A. McIntosh, E. R. Brown, K. B. Nichols, O. B. McMahon, W. F. DiNatale, and T. M. Lyszczarz, "Terahertz photomixing with diode lasers in low-temperature-grown GaAs," Applied Physics Letters, vol. 67, pp. 3844-3846, 1995.

[49] K. A. McIntosh, E. R. Brown, K. B. Nichols, O. B. McMahon, W. F. DiNatale, and T. M. Lyszczarz, "Terahertz measurements of resonant planar antennas coupled to low- 
temperature-grown GaAs photomixers," Applied Physics Letters, vol. 69, pp. 36323634, 1996.

[50] M. Tani, S. Matsuura, K. Sakai, and S. Nakashima, "Emission characteristics of photoconductive antennas based on low-temperature-grown GaAs and semi-insulating GaAs," Applied Optics, vol. 36, pp. 7853-7859, Oct 1997.

[51] E. R. Brown, "A photoconductive model for superior GaAs THz photomixers," Applied Physics Letters, vol. 75, pp. 769-771, 1999.

[52] M. Sukhotin, E. R. Brown, A. C. Gossard, D. Driscoll, M. Hanson, P. Maker, and R. Muller, "Photomixing and photoconductor measurements on ErAs/InGaAs at $1.55 \mathrm{mu}$ m," Applied Physics Letters, vol. 82, pp. 3116-3118, May 52003.

[53] R. Mendis, C. Sydlo, J. Sigmund, M. Feiginov, P. Meissner, and H. L. Hartnagel, "Tunable CW-THz system with a log-periodic photoconductive emitter," Solid-State Electronics, vol. 48, pp. 2041-2045, 2004.

[54] H. Roehle, R. J. B. Dietz, H. J. Hensel, J. Bottcher, H. Kunzel, D. Stanze, M. Schell, and B. Sartorius, "Next generation $1.5 \mathrm{mu} \mathrm{m}$ terahertz antennas: mesa-structuring of InGaAs/InAlAs photoconductive layers," Optics Express, vol. 18, pp. 2296-2301, 2010.

[55] S. Rihani, R. Faulks, H. E. Beere, I. Farrer, M. Evans, D. A. Ritchie, and M. Pepper, "Enhanced terahertz emission from a multilayered low temperature grown GaAs structure," Applied Physics Letters, vol. 96, 2010.

[56] B. Schulkin, X. C. Zhang, T. Tongue, J. Xu, and J. Chen, "Systems, Methods and Devices for Handling Terahertz Radiation," US20080239317, 2008.

[57] A. Hirano, H. Tsuda, H. Kobayashi, R. Takahashi, M. Asobe, K. Sato, and K. Hagimoto, "All-optical discrimination based on nonlinear transmittance of MQW semiconductor optical gates," Journal of Lightwave Technology, vol. 17, pp. 873-884, 1999.

[58] R. F. Kazarinov and R. A. Suris, "Possibility of Amplification of Electromagnetic Waves in a Semiconductor with a Superlattice," Soviet Physics Semiconductors-USSR, vol. 5, p. 707, 1971.

[59] J. L. Coutaz, J. F. Roux, A. Gaarder, S. Marcinkevicius, J. Jasinski, K. Korona, M. Kaminska, K. Bertulis, and A. Krotkus, "Be-doped low-temperature grown GaAs for ultrafast optoelectronic devices and applications," Simc-Xi: 2000 International Semiconducting and Insulating Materials Conference, Proceedings, pp. 89-96, 2000.

[60] E. Dupont, Z. R. Wasilewski, and H. C. Liu, "Terahertz emission in asymmetric quantum wells by frequency mixing of midinfrared waves," IEEE Journal of Quantum Electronics, vol. 42, pp. 1157-1174, Nov-Dec 2006.

[61] E. Rosencher, A. Fiore, B. Vinter, V. Berger, P. Bois, and J. Nagle, "Quantum engineering of optical nonlinearities," Science, vol. 271, pp. 168-173, Jan 121996.

[62] C. Sirtori, F. Capasso, J. Faist, L. N. Pfeiffer, and K. W. West, "Far-Infrared Generation by Doubly Resonant Difference-Frequency Mixing in a Coupled-Quantum-Well 2Dimensional Electron-Gas System," Applied Physics Letters, vol. 65, pp. 445-447, Jul 251994.

[63] F. Capasso, C. Sirtori, and A. Y. Cho, "Coupled-Quanntum-Well Semiconductors with Giant Electric-Field Tunable Nonlinear-Optical Properties in the Infrared," IEEE Journal of Quantum Electronics, vol. 30, pp. 1313-1326, May 1994.

[64] M. M. Fejer, S. J. B. Yoo, R. L. Byer, A. Harwit, and J. S. Harris, "Observation of Extremely Large Quadratic Susceptability at 9.6-10.8mu-m in Electric-Field-Biased AlGaAs Quantum Wells," Physical Review Letters, vol. 62, pp. 1041-1044, Feb 27 1989.

[65] M. A. Belkin, F. Capasso, F. Xie, A. Belyanin, M. Fischer, A. Wittmann, and J. Faist, "Room temperature terahertz quantum cascade laser source based on intracavity difference-frequency generation," Applied Physics Letters, vol. 92, 201101, May 19 2008.

[66] J. Faist, M. Beck, T. Aellen, and E. Gini, "Quantum-cascade lasers based on a boundto-continuum transition," Applied Physics Letters, vol. 78, pp. 147-149, 2001. 
[67] S. Barbieri, "Terahertz Quantum Cascade Laser," GB2409104, 2005.

[68] M. Lee and M. C. Wanke, "Integrated Heterodyne Terahertz Transceiver," US7550734, 2009.

[69] M. Asada and S. Suzuki, "Terahertz oscillators using electron devices - an approach with Resonant tunneling diodes," IEICE Electronics Express, vol. 8, pp. 1110-1126, 2011.

[70] M. Asada, S. Suzuki, and N. Kishimoto, "Resonant Tunneling Diodes for Sub-Terahertz and Terahertz Oscillators," Japanese Journal of Applied Physics, vol. 47, p. 4375, 2008.

[71] S. Hiroki, Y. Haruki, A. Masahiro, and S. Sukefumi, "Resonance Tunnel Diode and Terahertz Oscillator," Japan Patent JP2011114120, 2011.

[72] S. Suzuki, M. Asada, A. Teranishi, H. Sugiyama, and H. Yokoyama, "Fundamental oscillation of resonant tunneling diodes above $1 \mathrm{THz}$ at room temperature," Applied Physics Letters, vol. 97, 242102, Dec 132010.

[73] H. Ito, T. Furuta, S. Kodama, and T. Ishibashi, "InP/lnGaAs uni-travelling-carrier photodiode with 310GHz bandwidth," Electronics Letters, vol. 36, pp. 1809-1810, 2000.

[74] H. Ito, T. Furuta, F. Nakajima, K. Yoshino, and T. Ishibashi, "Photonic generation of continuous $\mathrm{THz}$ wave using uni-traveling-carrier photodiode," Lightwave Technology, Journal of, vol. 23, pp. 4016-4021, 2005.

[75] S. Matsuura, M. Tani, and K. Sakai, "Generation of coherent terahertz radiation by photomixing in dipole photoconductive antennas," Applied Physics Letters, vol. 70, pp. 559-561, 1997.

[76] P. R. Smith, "Subpicosecond photoconducting dipole antennas," IEEE Journal of Quantum Electronics, vol. 24, p. 255, 1988.

[77] P. R. Smith, D. H. Auston, and M. C. Nuss, "Subpicosecond Photoconducting Dipole Antennas," IEEE Journal of Quantum Electronics, vol. 24, pp. 255-260, 1988.

[78] J. T. Darrow, B. B. Hu, X. C. Zhang, and D. H. Auston, "Subpicosecond Electromagnetic Pulses from Large-Aperture Photoconducting Antennas," Optics Letters, vol. 15, pp. 323-325, 1990.

[79] E. R. Brown, Advancements in photomixing and photoconductive switching for $\mathrm{THz}$ spectroscopy and imaging vol. 7938: SPIE, 2011.

[80] C. L. Wang and C. L. Pan, "Tunable Multiterahertz Beat Signal Generation from a 2Wavelength Laser-Diode Array," Optics Letters, vol. 20, pp. 1292-1294, 1995.

[81] T. Hidaka, S. Matsuura, M. Tani, and K. Sakai, "CW terahertz wave generation by photomixing using a two-longitudinal-mode laser diode," Electronics Letters, vol. 33, pp. 2039-2040, 1997.

[82] C. S. Friedrich, C. Brenner, S. Hoffmann, A. Schmitz, I. C. Mayorga, A. Klehr, G. Erbert, and M. R. Hofmann, "New two-color laser concepts for THz generation," IEEE Journal of Selected Topics in Quantum Electronics, vol. 14, pp. 270-276, 2008.

[83] S. A. Zolotovskayaa and N. Daghestani, "Stable dual-wavelength operation of InGaAs diode lasers with volume Bragg gratings," Applied Physics Letters, vol. 91, p. 171113, 2007.

[84] S. A. Zolotovskaya, V. I. Smirnov, G. B. Venus, L. B. Glebov, and E. U. Rafailov, "Two-Color Output From InGaAs Laser With Multiplexed Reflective Bragg Mirror," IEEE Photonics Technology Letters, vol. 21, pp. 1093-1095, 2009.

[85] F. W. Smith, A. R. Calawa, C. L. Chen, M. J. Manfra, and L. J. Mahoney, "New MBE Buffer Used to Eliminate Backgating in GaAs-MESFETs," IEEE Electron Device Letters, vol. 9, pp. 77-80, 1988.

[86] D. C. Look, "Molecular-Beam Epitaxial GaAs Grown at Low-Temperatures," Thin Solid Films, vol. 231, pp. 61-73, 1993.

[87] S. Gupta, J. F. Whitaker, and G. A. Mourou, "Ultrafast Carrier Dynamics in III-IV Semiconductors Grown by Molecular-Beam Epitaxy at Very Low Substrate Temperatures," IEEE Journal of Quantum Electronics, vol. 28, pp. 2464-2472, 1992. 
[88] F. W. Smith, H. Q. Le, V. Diadiuk, M. A. Hollis, A. R. Calawa, S. Gupta, M. Frankel, D. R. Dykaar, G. A. Mourou, and T. Y. Hsiang, "Picosecond Gaas-Based Photoconductive Optoelectronic Detectors," Applied Physics Letters, vol. 54, pp. 890892, Mar 61989.

[89] E. Estacio, "Strong enhancement of terahertz emission from GaAs in InAs/GaAs quantum dot structures," Applied Physics Letters, vol. 94, p. 232104, 2009.

[90] V. Pacebutas, K. Bertulis, A. Biciunas, and A. Krotkus, "Low-temperature MBE-grown GaBiAs layers for terahertz optoelectronic applications," Physica Status Solidi C Current Topics in Solid State Physics, Vol 6, No 12, vol. 6, pp. 2649-2651, 2009.

[91] J. B. D. Soole and H. Schumacher, "Transit-Time Limited Frequency Response of InGaAs MSM Photodetectors," IEEE Transactions on Electron Devices, vol. 37, pp. 2285-2291, 1990.

[92] B. Grandidier, H. J. Chen, R. M. Feenstra, D. T. McInturff, P. W. Juodawlkis, and S. E. Ralph, "Scanning tunneling microscopy and spectroscopy of arsenic antisites in low temperature grown InGaAs," Applied Physics Letters, vol. 74, pp. 1439-1441, Mar 8 1999.

[93] C. Baker, I. S. Gregory, W. R. Tribe, I. V. Bradley, M. J. Evans, E. H. Linfield, and M. Missous, "Highly resistive annealed low-temperature-grown InGaAs with sub-500 fs carrier lifetimes," Applied Physics Letters, vol. 85, pp. 4965-4967, Nov 222004.

[94] C. Baker, I. Gregory, M. Evans, W. Tribe, E. Linfield, and M. Missous, "Alloptoelectronic terahertz system using low-temperature-grown InGaAs photomixers," Opt. Express, vol. 13, pp. 9639-9644, 2005.

[95] M. P. Hanson, D. C. Driscoll, E. Muller, and A. C. Gossard, "Microstructure and electronic characterization of InGaAs containing layers of self-assembled ErAs nanoparticles," Physica E: Low-dimensional Systems and Nanostructures, vol. 13, pp. 602-605, 2002.

[96] M. Griebel, J. H. Smet, D. C. Driscoll, J. Kuhl, C. A. Diez, N. Freytag, C. Kadow, A. C. Gossard, and K. Von Klitzing, "Tunable subpicosecond optoelectronic transduction in superlattices of self-assembled ErAs nanoislands," Nature Materials, vol. 2, pp. 122126, 2003.

[97] C. D. Wood, O. Hatem, J. E. Cunningham, E. H. Linfield, A. G. Davies, P. J. Cannard, M. J. Robertson, and D. G. Moodie, "Terahertz emission from metal-organic chemical vapor deposition grown Fe:InGaAs using $830 \mathrm{~nm}$ to $1.55 \mathrm{mu}$ m excitation," Applied Physics Letters, vol. 96, p. 194104, 2010.

[98] S. Verghese, K. A. McIntosh, and E. R. Brown, "Highly tunable fiber-coupled photomixers with coherent terahertz output power," IEEE Transactions on Microwave Theory and Techniques, vol. 45, pp. 1301-1309, 1997.

[99] A. Jackson, "Low-Temperature-Grown GaAs Photomixers Designed for Increased Terahertz Output Power," Ph.D. thesis, Univ. California, Santa Barbara, 1999.

[100] J. Darmo, T. Muller, G. Strasser, K. Unterrainer, and G. Tempea, "Terahertz emitter with integrated semiconductor Bragg mirror," Electronics Letters, vol. 39, pp. 460-462, 2003.

[101] R. Faulks, "Enhanced Terahertz Receiver Using a Distributed Bragg Reflector Coupled to a Photoconductive Antenna," IEEE Photonics Technology Letters, vol. 21, p. 1603, 2009.

[102] C. Sydlo, "Characterisation of a circularly-toothed planar logarithmic-periodic antenna for broadband power detection," Frequenz, vol. 58, p. 207, 2004.

[103] R. Mendis, C. Sydlo, J. Sigmund, M. Feiginov, P. Meissner, and H. L. Hartnagel, "Spectral characterization of broadband $\mathrm{THz}$ antennas by photoconductive mixing: Toward optimal antenna design," IEEE Antennas and Wireless Propagation Letters, vol. 4, pp. 85-88, 20052005.

[104] S. M. Duffy, S. Verghese, K. A. McIntosh, A. Jackson, A. C. Gossard, and S. Matsuura, "Accurate modeling of dual dipole and slot elements used with photomixers for coherent terahertz output power," IEEE Transactions on Microwave Theory and Techniques, vol. 49, pp. 1032-1038, 2001. 
[105] M. Naftaly, P. Dean, R. E. Miles, J. R. Fletcher, and A. Malcoci, "A Simple Interferometer for the Analysis of Terahertz Sources and Detectors," IEEE Journal of Selected Topics in Quantum Electronics, vol. 14, pp. 443-448, 2008.

[106] Q. M. C. Instruments. Available: http://www.terahertz.co.uk/index.php?option=com_content\&view=article\&id=211\&Ite $\underline{\operatorname{mid}=538}$

[107] Tydex. Available: http://www.tydexoptics.com/en/products/thz_optics/thz_lens/ 